\title{
Podiatric medical care: an often neglected part of the health care received by older frequently at risk individuals
}

\section{Commentary}

The prevalence of foot problems is considerably greater among older people as a result of the chronic disorders that this age group is more likely to have causing symptoms in the feet which may result in disabling pain, infection, and amputation.. Among these are such conditions as osteoarthritis, peripheral vascular disorders, diabetes, and neurological conditions.

For example, type 2 diabetes in the U.S affects more than one of every four people (25.9percent) 65years of age and over and the importance of foot care in that population cannot be overestimated. Among the major complications of diabetes is lower extremity amputation. The American Diabetes Association reports that in 2010, about 73,000 non-traumatic lower-limb amputations were performed in adults aged 20 years or older with diagnosed diabetes. About $60 \%$ of non-traumatic lower-limb amputations among people aged 20years or older occur in people with diabetes. Except for trauma diabetes is the most frequent cause of lower limb amputation. ${ }^{1}$ Furthermore victims of lower limb amputation have a significantly reduced five year survival rate. Of persons with diabetes who have a lower extremity amputation, up to $55 \%$ will require amputation of the second leg within 2-3years. ${ }^{2}$

In spite of this, relatively little attention is paid to the foot in routine physical examinations and many people provide potentially dangerous self-care to their feet including such intervention as cutting corns and calluses and digging out "in-growing toenails" since they consider such procedures innocuous. Unfortunately those who engage in such "bathroom surgery" are at risk of developing infection which in those with diabetes and/or with diminished blood circulation, are prone to infection as well as amputation of all or part of their lower extremity. Others use "corn pads" that include salicylic acid which, while removing the callus material, may also destroy underlying normal skin putting people at risk of developing infection and/or skin ulceration. This in a person with diminished blood supply and/ or diabetes can lead to amputation. Primary care physicians too often pay little attention to the foot of patients with diabetes and/or diminished circulation. Fortunately many infections and amputations can be prevented through preventive care consisting of a combination of patient education in foot care and podiatric medical care which includes a foot examination beginning after diabetes and/or inadequate circulation is diagnosed. After such an assessment periodic foot examinations should be provided every two to three months or more frequently as appropriate to the patient's condition. Also rarely cited or underemphasized by health care providers is the effect on the cardiovascular system of foot disorders. That is, while patients are frequently advised by their primary care physician to exercise, many find this difficult to do if they have disorders that result in painful feet restricting their mobility.

Podiatric medical practice also focuses on the medical and surgical care of foot injuries such as fractures and dislocations as well
Volume I Issue 2 - 2017

\author{
Leonard A Levy \\ Professor of Family Medicine/ Public Health/ Biomedical \\ Informatics, Nova Southeastern University, USA
}

\begin{abstract}
Correspondence: Leonard A Levy, Professor of Family Medicine/ Public Health/ Biomedical Informatics, Nova Southeastern University, USA, Fort Lauderdale, FL 33328-2018, Tel+ 954.262.1469, Email levyleon@nova.edu
\end{abstract}

Received: March 29, 2017 | Published: April 05, 2017

as congenitally caused foot and ankle deformities. Skin and nerve conditions affecting the pedal extremity also are a part of podiatric practice. $^{3}$

\section{Education and training for podiatric medical practice}

The podiatric physician is a highly trained health care professional who completes a premedical college education equivalent to that of those who enter professional school to acquire the MD or DO degree and also are required to complete the Medical College Admissions Test (MCAT). Those preparing for the practice of podiatric medicine then complete a four year professional school curriculum leading to the degree of Doctor of Podiatric Medicine (DPM) followed by a hospitalbased residency of at least three additional years. Upon completing their education and training and acquiring a license to practice, they enter clinical practice that may be hospital or community practice based or as a commissioned officer in the military beginning at the rank of captain. Upon completion of residency training, the DPM is eligible to sit for an examination leading to board certification. The license to practice podiatric medicine and surgery permits the prevention, diagnosis, medical, surgical, and rehabilitative care of problems affecting the foot and ankle. This includes the prescription and use of oral, topical, and injectible agents such as but not limited to antibiotics, anti-inflammatory drugs, narcotic and anesthetic agents, as well as x-ray and other imaging modalities, and devices to accommodate and treat foot deformities. The DPM also is permitted to admit patients to accredited hospitals for medical and surgical foot care including the performance of soft tissue and bone surgery.

\section{Acknowledgements}

None.

\section{Conflict of interest}

Author declares there is no conflict of interest in publishing the article. 


\section{References}

1. http://www.diabetes.org/diabetes basics/statistics/\#sthash.udfuwTyi. dpuf
2. Limb Loss Statistics. Amputee Coalition, New York, USA; 2017.

3. National Institute of Neurological Disorders and Stroke. Peripheral Neuropathy Information Page Accessed 7/29/2014, USA; 2014 